\title{
Dispersion Of Group Judgments
}

\author{
Thomas L. Saaty, Luis G. Vargas* \\ The Joseph M. Katz Graduate School of Business, University of Pittsburgh, Pittsburgh, PA 1560 USA
}

\begin{abstract}
To achieve a decision with which the group is satisfied, the group members must accept the judgments, and ultimately the priorities. This requires that (a) the judgments be homogeneous, and (b) the priorities of the individual group members be compatible with the group priorities. There are three levels in which the homogeneity of group preference needs to be considered: (1) for a single paired comparison (monogeneity), (2) for an entire matrix of paired comparisons (multigeneity), and (3) for a hierarchy or network (omnigeneity). In this paper we study monogeneity and the impact it has on group priorities.
\end{abstract}

Keywords: Reciprocal uniform distribution, Geometric mean, Geometric dispersion, Group cohesiveness, Group liaison, Principal right eigenvector, Beta distribution

\section{Introduction}

In all facets of life groups of people get together to make decisions. The group members may or may not be in agreement about some issues and that is reflected in how homogeneous the group is in its thinking. In the AHP groups make decisions by building a hierarchy together and providing judgments expressed on a 1 to 9 discrete scale having the reciprocal property. Condon et al. [1] mentioned that there are four different ways in which groups estimate weights in the AHP: “...consensus, vote or compromise, geometric mean of the individual judgments, and weighted arithmetic mean." The first three deal with judgments of individuals while the last deals with the priorities derived from the judgments.

To achieve a decision with which the group is satisfied, the judgments, and ultimately the priorities, must be accepted by the group members. This requires that (a) the judgments be homogeneous, and (b) the priorities of the individual group members be compatible with the group priorities.

There are three levels in which the homogeneity of group preference needs to be considered: (1) for a single paired comparison (monogeneity), (2) for an entire matrix of paired comparisons (multigeneity), and (3) for a hierarchy or network (omnigeneity). Monogeneity relates to the dispersion of the judgments around their geometric mean. The geometric mean of group judgments is the mathematical equivalent of consensus if all the members are considered equal. Otherwise one would use the weighted geometric mean. Aczel and Saaty [2] showed that the only mathematically valid way to synthesize reciprocal judgments preserving the reciprocal condition is the geometric mean. If the group judgments for a single paired comparison are too dispersed, i.e., they are not close to their geometric mean, the resulting geometric mean may not be used as the representative judgment for the group.

\footnotetext{
* Corresponding author

E-mail Addresses: saaty@katz.pitt.edu (T.L. Saaty), lgvargas@pitt.edu (L.G. Vargas)
} 
Multigeneity relates to the compatibility index of the priority vectors. The closeness of two priority vectors $v=\left(v_{1}, \ldots, v_{n}\right)^{T}$ and $w=\left(w_{1}, \ldots, w_{n}\right)^{T}$ can be tested through their compatibility index [3] given by $\frac{1}{n^{2}} e^{T} V \circ W^{T} e$, where $\circ$ is the Hadamard or elementwise product, $V=\left(v_{i} / v_{j}\right)$ and $W=\left(w_{i} / w_{j}\right)$. Note that for a reciprocal matrix $A=\left(a_{i j}\right)$ with principal eigenvalue $\lambda_{\max }$ and corresponding right eigenvector $w=\left(w_{1}, \ldots, w_{n}\right)$, $\frac{1}{n^{2}} e^{T} A \circ W^{T} e=\lambda_{\max } / n^{2}$. Thus, one can test the compatibility of each individual vector with that derived from the group judgments. A homogeneous group should have compatible individuals. It is clear that homogeneity at the paired comparisons level implies compatibility at the group level, but the converse is not always true. At the hierarchy or network level, it appears that it is more meaningful to speak of compatibility than of homogeneity. The main thrust of this paper is to study monogeneity.

Dispersion in judgments leads to violations of Pareto Optimality at both the pairwise comparison level and/or the entire matrix from which priorities are derived. Ramanathan and Ganesh [4] explored two methods of combining judgments in hierarchies but they violated the Pareto Optimality Principle for pairwise comparisons [5], and hence, they incorrectly concluded that the geometric mean violates Pareto Optimality. Pareto Optimality at the pairwise level is not sufficient to ensure Pareto Optimality at the priority level. Fundamentally, Pareto Optimality means that if all individuals prefer A to B then so should the group. The group may be homogeneous in some paired comparisons and heterogeneous in others thus violating Pareto Optimality. The degree of violation of Pareto Optimality can be measured by computing compatibility along the rows, which yields a vector of compatibility values. What does one do when a group is not homogeneous in all its comparisons? Lack of homogeneity (heterogeneity) on some issues may lead to breaking up the group into smaller homogeneous groups. How should one separate the group into homogeneous subgroups? Since homogeneity relates to dispersion around the geometric mean, and dispersion itself involves uncertainties, how much of the dispersion is innate and how much is noise that when filtered one can speak of true homogeneity? In other words, how does one separate random considerations from committed beliefs?

Dispersion at the single paired comparison level affects the priorities obtained by each group member individually and could lead to violating Pareto Optimality. Should one combine or synthesize the priorities of the individuals to obtain the group priority or should one combine their judgments?

Here we develop a way to test monogeneity, i.e., how homogeneous the judgments of the members of a group are for each judgment they give in response to paired comparisons. This is done by deriving a measure of the dispersion of the judgments based on the geometric mean. Computing the dispersion around the geometric mean requires a multiplicative approach rather than the usual additive expected value used to calculate moments around the arithmetic mean. This leads to a new multiplicative or geometric expected value used to define the concept of geometric dispersion. The geometric dispersion of a finite set of values is given by the geometric mean of the ratios of the values to their geometric mean, if the ratio is greater than 1 , or the reciprocal, if the ratio is less than or equal to 1 . This measure of variability or dispersion of the judgments around the geometric mean allows us to (a) determine if the 
geometric mean of the judgments of a group can be used as the synthesized group judgment, (b) if the geometric mean cannot be used, divide the group into subgroups according to their geometric dispersion, and (c) measure the variability of the priorities corresponding to the matrix of judgments synthesized for the group.

In general, unless a group decides through consensus which judgments to assign in response to a paired comparison, the individual members may give different judgments. We need to find if the dispersion of this set of judgments is a normal occurrence in the group behavior. To do this, we compare the dispersion of the group with the dispersion of a group providing random responses to the paired comparison. Thus, we assume that an individual's pairwise comparison judgments about homogeneous elements is considered random, and expressed on a discrete $1 / 9, \ldots, 1 / 2,1,2, \ldots, 9$ scale of seventeen equally likely values. A sample consists of a set of values selected at random from the set of seventeen values, one for each member of the group. It is the dispersion of this sample of numbers around its geometric mean that concerns us. This dispersion can be considered a random variable with a distribution. Because treating the judgments as discrete variables becomes an intractable computational problem as the group size increases, we assume that judgments belong to a continuous random distribution. For example, if there are five people each choosing one of 17 numbers in the scale $1 / 9, \ldots, 1, \ldots, 9$, there are $17^{5}=1,419,857$ possible combinations of which 20,417 are different. Thus, the dispersion of each sample from its geometric mean has a large number of values for which one needs to determine the frequency and thus the probability distribution. To deal with this complexity, we use the continuous generalization instead. This allows us to fit probability distributions to the geometric dispersion for groups of arbitrary size. Once we have the continuous distribution of the geometric dispersion, the parameters that characterize this distribution are a function of the number of individuals $n$ in the group.

To use the geometric mean to synthesize a set of judgments given by several individuals in response to a single pairwise comparison, as the representative judgment for the entire group, the dispersion of the set of judgments from the geometric mean must be within some prescribed bounds. To determine these bounds, we use the probability distribution of the sample geometric dispersion mentioned above. We can then find how likely the observed value of the sample geometric dispersion is. This is done by computing the cumulative probability below the observed value of the sample dispersion in the theoretical distribution of the dispersion. If it is small then the observed value is less likely to be random, and we can then infer that the geometric dispersion of the group is "small" and the judgments can be considered homogeneous or $\alpha$-cohesive at that specified $\alpha$ level. On the other hand, if the dispersion is unacceptable, then we could divide the group of individuals into subgroups representing similarity in judgment.

The remainder of the paper is structured as follows. In section 2 we give a summary of the geometric expected value concept and its generalization to the continuous case that leads to the concept of product integral. In section 3 we define the geometric dispersion of a positive random variable and apply it to the judgments of groups. In section 4 we approximate the distribution of the group geometric dispersion. In section 5 we sketch how groups could be divided into subgroups if the geometric dispersion is 
large, and in section 6 we show the impact of the dispersion of a group's judgments on the priorities associated with their judgments.

\section{Generalization of the Geometric Mean to the Continuous Case}

Let $X$ be a random variable. Given a sample from this random variable $\tilde{X}=\left(x_{1}, \ldots, x_{n}\right)$, the sample geometric mean is given by $\bar{x}_{G} \equiv \prod_{i=1}^{n} x_{i}^{1 / n}$. Let us assume that not all the values are equally likely, and their absolute frequencies are equal to $m_{1}, \ldots, m_{k}$ with $\sum_{i=1}^{k} m_{i}=n$. Then, the sample geometric mean is given by: $\bar{x}_{G} \equiv\left[\prod_{i=1}^{k} x_{i}^{m_{i}}\right]^{1 / n}=\prod_{i=1}^{k} x_{i}^{m_{i} / n}$. An estimate of the probabilities $p_{i}=P\left[X=x_{i}\right]$ is given by $\hat{p}_{i}=\frac{m_{i}}{n}$. Thus the geometric expected value of a discrete random variable $X$ is given by:

$$
E_{G}[X]=\prod_{\forall x_{i}} x_{i}^{P\left[X=x_{i}\right]}=e^{\left\{\sum_{\forall x_{i}}^{P\left[X=x_{i}\right] \ln x_{i}}\right\}}=e^{E[\ln X]}
$$

In the continuous case, because $P[X=x]=0$ for all $x$, we need to use intervals rather than points, and hence, we obtain:

$$
E_{G}[X]=\lim _{\Delta x \rightarrow 0} \prod_{\forall x} x^{P[x<X \leq x+\Delta x]}=\prod_{\forall x} x^{f(x) d x}
$$

Equation (2) is known as the product integral [6]. If $X$ is defined in the interval $(s, t]$, we have

$$
\ln E_{G}[X]=\lim _{\Delta x \rightarrow 0} \sum_{s \leq x \leq t} P[x<X \leq x+\Delta x] \ln x_{i}=\int_{(s, t]} f(x) \ln x d x .
$$

In general, we have

$$
E_{G}[X]=\prod_{D(X)} x^{f(x) d x}=e^{\int_{D(X)} f(x) \ln x d x}=\mathrm{e}^{\{E[\ln X]\}}
$$

where $\mathrm{D}(X)$ is the domain of the variable $X$ and $\int_{\mathrm{D}(X)} f(x) d x=1$.

\section{The Geometric Dispersion of a Positive Random Variable}

Using the geometric expected value, we define a measure of dispersion similar to the standard deviation. Let $\sigma_{G}$ be the geometric dispersion of a positive random variable $X$ given by $\sigma_{G}(X)=E_{G}\left[\left|\frac{X}{\mu_{G}}\right|_{G}\right]$, where $|x|_{G}=\left\{\begin{array}{lll}x & \text { if } & x>1 \\ \frac{1}{x} & \text { if } & x \leq 1\end{array}\right.$. For $|\ln x|=\left\{\begin{array}{ll}\ln x, & x>1 \\ \ln \frac{1}{x}, & x \leq 1\end{array}\right.$, then $e^{\ln x \mid}=\left\{\begin{array}{ll}x, & x>1 \\ \frac{1}{x}, & x \leq 1\end{array}\right.$ and $\quad \sigma_{G}(X)=\exp \left\{E\left[\mid \ln \frac{x}{\mu_{G}}\right\}\right\}=\mu_{G}{ }^{2 F\left(\mu_{G}\right)} \exp \left\{-2 \int_{0}^{\mu_{G}}(\ln x) f(x) d x\right\} . \quad$ It is possible now to write $x=\mu_{G} \omega^{\sigma_{G}}$, where the variable $\omega$ has a geometric mean equal to 1 and a geometric dispersion equal to $e^{-2 \int_{0}^{1} \ln x f(x) d x}$.

Let $X_{k}, k=1,2, \ldots, n$ be the independent identically distributed random variables associated with the judgments. Let $\left\{X_{k}, k=1,2, \ldots, n\right\}$ be continuous random variables distributed according to a reciprocal uniform $R U\left[\frac{1}{9}, 9\right]$, i.e., the variable $Y_{k}=\ln X_{k}$ is a 
uniform random variable defined in the interval $[-\ln 9, \ln 9]$. The probability density function (pdf) of $Y_{k}$ is given by $g(y)=\frac{1}{2 \ln 9} I_{[-\ln 9, \ln 9]}(y)$, and hence, the pdf of $X_{k}$ is given by $f(x)=\frac{1}{2 \ln 9} \frac{1}{x} I_{\left[\frac{1}{9}, 9\right]}(x)$.

The sample geometric dispersion is given by:

$$
s_{G}\left(x_{1}, \ldots, x_{n}\right)=\left[\prod_{k=1}^{n}\left|\frac{x_{k}}{\bar{x}_{G}}\right|_{G}\right]^{1 / n}=\left[\prod_{k=1}^{n} e^{\mid \ln \frac{x_{k}}{\bar{x}_{G}}}\right]^{1 / n}
$$

Let $\left(x_{[1: n]}, \ldots, x_{[n: n]}\right)$ be the order statistics corresponding to the sample $\left\{x_{k}, k=1,2, \ldots, n\right\}$, i.e., $x_{[h: n]} \leq x_{[k: n]}$ if $h \leq k$. Let $n_{1}$ be a value for which $x_{[k: n]} \leq \bar{x}_{G}$ for $k=1,2, \ldots, n_{1}$. We have

$$
\ln s_{G}\left(x_{[1: n]}, \ldots, x_{[n: n]}\right)=\frac{1}{n} \sum_{k=1}^{n}\left|\ln \frac{x_{[k: n]}}{\bar{x}_{G}}\right|=\frac{2 n_{1}}{n}\left[\ln \bar{x}_{G}-\ln \bar{x}_{\left[n_{1}: n\right]}^{G}\right]
$$

and hence, we obtain

$$
s_{G}\left(x_{1}, \ldots, x_{n}\right)=s_{G}\left(x_{[1: n]}, \ldots, x_{[n: n]}\right)=\left(\bar{x}_{G} / \bar{x}_{\left[n_{1}: n\right]}^{G}\right)^{2 n_{1} / n} \text {. }
$$

For a group consisting of $n$ individuals, the distribution of $S_{G}\left(X_{1}, \ldots, X_{n}\right)$ is given by

$$
P\left[S_{G} \leq s\right]=\sum_{n_{1}=1}^{n} P\left[\left[\frac{\bar{X}_{G}}{\bar{X}_{\left[n_{1}: n\right]}^{G}}\right]^{2 n_{1} / n} \leq s \mid v_{n}=n_{1}\right] P\left[v_{n}=n_{1}\right]
$$

where $v_{n}=v_{n}\left(A, \bar{x}_{G}\right)$ represents the number of occurrences of the event $A \equiv\left\{X_{k} \leq \bar{x}_{G}\right\}$, and it is also equal to the index of the largest order statistic less than or equal to the sample geometric mean [7]. Let $S_{k, n}\left(\bar{x}_{G}\right)=\sum_{1 \leq i_{1}<i_{2}<\cdots<i_{k} \leq n} P\left[X_{i_{1}} \geq \bar{x}_{G}, X_{i_{2}} \geq \bar{x}_{G}, \ldots, X_{i_{k}} \geq \bar{x}_{G}\right]$. Since $S_{k, n}\left(\bar{x}_{G}\right)=\sum_{r=k}^{n}\left(\begin{array}{l}r \\ k\end{array}\right) P\left[v_{n}=r\right]$, and $P\left[v_{n}=r\right]=\sum_{k=0}^{n-r}(-1)^{k}\left(\begin{array}{c}k+r \\ r\end{array}\right) S_{k+r, n}$ we have

$$
P\left[S_{G} \leq s\right]=\sum_{t=1}^{n} P\left[\left[\frac{\bar{X}_{G}}{\bar{X}_{[t: n]}^{G}}\right]^{2 t / n} \leq s \mid v_{n}=t\right] \sum_{k=0}^{n-t}(-1)^{k}\left(\begin{array}{c}
k+t \\
t
\end{array}\right) S_{k+t, n} .
$$

Thus, the density function is given by:

$$
f_{G D}(s)=\sum_{t=1}^{n} f_{G D}(s \mid t) \sum_{k=0}^{n-t}(-1)^{k}\left(\begin{array}{c}
k+t \\
t
\end{array}\right) S_{k+t, n}
$$

that is a convex combination of density functions of variables of the form $\left(\prod_{k=1}^{n}\left(X_{k}\right)^{1 / n} / \prod_{h=1}^{n_{1}}\left(X_{h}\right)^{1 / n_{1}}\right)^{\frac{2 n_{1}}{n}}$, i.e., the ratio of products of reciprocal uniform variates. These density functions are of the form $\frac{1}{z}\left(a_{0}+a_{1} \ln [z]+\cdots+a_{n-1} \ln [z]^{n-1}\right)$.

There are closed form expressions for the density function of the geometric dispersion for a group consisting of three or less individuals, but for groups larger than three, it is cumbersome and not much precision is gained from it. Instead, we approximate them using simulation. 


\section{Approximations of the Geometric Dispersion of Group Judgments}

We computed the geometric dispersion of randomly generated samples of size 20,000 under the assumption that the judgments are distributed according to a continuous reciprocal uniform distribution $R U\left[\frac{1}{9}, 9\right]$. We did this for groups consisting of $4,5, \ldots, 15,20,25,30,35,40,45$, and 50 individuals. We found that as the group size increases, the geometric dispersion tends to become gamma distributed. The parameters of these gamma distributions with location parameter equal to 1 are given in Table 1. To extend these models to groups of any size, we fit regression models to the parameters of the gamma distributions. Regression models of the shape $(\alpha)$ and the scale $(\beta)$ parameters versus $n$ appear to be surprisingly robust:

$$
\begin{array}{ll}
\alpha(\text { shape })=-3.48226+1.40829 * n & (\text { R-squared }=99.9741) \\
\beta(\text { scale })=0.897865+0.504361 * n & (\text { R-squared }=99.981)
\end{array}
$$

In addition, the average and variance of the geometric dispersion can also be estimated from the parameters of these models:

$$
\begin{array}{cc}
\text { mean }=\exp (1.03505-1.01298 / n) & (\mathrm{R} \text {-squared }=99.8463) \\
\text { variance }=7.23275 * n^{-1.0664} & (\mathrm{R} \text {-squared }=99.9706)
\end{array}
$$

Note that as $n$ tends to infinity, the average geometric dispersion tends to 2.81524 (99\% C.I. (2.79228,2.8384)) and the variance tends to zero (99\% C.I. (1.44E-9, 2.31E-9)).

We now have the basis for a statistical test to decide if the dispersion of a group can be considered larger than usual, i.e., that the probability of obtaining the value of the sample geometric dispersion of the group is greater than a pre-specified significance level (e.g., 5 percent) in the distribution of the group geometric dispersion. For example, for a group of size 6 , whose judgments on a given issue are equal to [2 9, 1, 2], the geometric dispersion of the group is equal to 1.9052169. The average geometric dispersion is estimated to be equal to $\exp (1.03505-1.01298 / 6)=2.378$. Taking the usual significance level of 5 percent, we observe that $P\left[S_{G}(6)<1.9052169\right]=0.0376176<0.05$. Thus, the $p$-value corresponding to the sample geometric dispersion indicates that it seems rare to observe values of the geometric dispersion smaller than the sample geometric dispersion, and hence, the geometric dispersion of the group is not unusually large, which in turn implies that the geometric mean can be used as the representative preference judgment for the entire group.

\section{Group Member Classification by the Geometric Dispersion}

Let us assume that $\left\{x_{k}, k=1,2, \ldots, n\right\}$ is a group of judgments and let $\left\{x_{[k: n]}, k=1,2, \ldots, n\right\}$ be their order statistics. If $F_{G D}\left[s_{G}\left(x_{1}, \ldots, x_{n}\right)\right] \equiv P\left[S_{G}\left(X_{1}, \ldots, X_{n}\right) \leq s_{G}\left(x_{1}, \ldots, x_{n}\right)\right]<\alpha$ (where $\alpha$ is usually taken to be equal to 0.05 ) then the geometric mean can be used as a representative of the group judgment. On the other hand, if $F_{G D}\left[s_{G}\left(x_{1}, \ldots, x_{n}\right)\right] \equiv P\left[S_{G}\left(X_{1}, \ldots, X_{n}\right) \leq s_{G}\left(x_{1}, \ldots, x_{n}\right)\right]>\alpha$ then the group needs to discuss the paired comparisons further in an attempt to reach consensus. To determine which members of a group disagree the most and hence make the geometric dispersion large, we find the p-values corresponding to the geometric dispersions of the groups of judgments given by: $\left\{x_{[1: n]}, x_{[2: n]}\right\}, \ldots,\left\{x_{[1: n]}, x_{[2: n]}, \ldots, x_{[k: n]}\right\}, \ldots,\left\{x_{[1: n]}, x_{[2: n]}, \ldots, x_{[n: n]}\right\}$. 
Let $s_{G}(k)=s_{G}\left(x_{[1: n]}, \ldots, x_{[k: n]}\right), k=2, \ldots, n$. We give without proof because of space limitations the following results.

Lemma 1: $s_{G}(k)=s_{G}\left(x_{[1: n]}, \ldots, x_{[k: n]}\right)$ is a non-decreasing function of $k$, i.e., $s_{G}(k) \geq s_{G}(k-1)$.

Theorem 1: Given a set of judgments $\left\{x_{[k: n]}, k=1,2, \ldots, n\right\}$ with corresponding ordered geometric dispersions $\left\{s_{G}(k), k=1,2, \ldots, n\right\}$, if for any $k, P\left[S_{G}(k) \leq s_{G}(k)\right] \leq \alpha$ then $P\left[S_{G}(k-1) \leq s_{G}(k-1)\right] \leq \alpha$.

Definition: A group of judgments $\left\{x_{k}, k=1,2, \ldots, n\right\}$ is said to be $\alpha$-cohesive if $P\left[S_{G}(n) \leq s_{G}(n)\right] \leq \alpha$.

Definition: A member of a group of $\alpha$-cohesive judgments is said to be a liaison of the group if the group is not $\alpha$-cohesive after the elimination of the corresponding judgment from the set of judgments.

\section{Table 1}

Gamma Distribution Parameters $(\gamma=1) \operatorname{Gamma}(\alpha, \beta, \gamma)=\frac{\beta^{\alpha}}{\Gamma(\alpha)}(x-\gamma)^{\alpha-1} e^{-\beta(x-\gamma)}$

\begin{tabular}{|c|r|r|}
\hline $\mathrm{n}$ & $\begin{array}{c}\text { Shape } \\
\alpha\end{array}$ & $\begin{array}{c}\text { Scale } \\
\beta\end{array}$ \\
\hline 4 & 2.80051 & 1.27561 \\
5 & 4.03976 & 1.76548 \\
6 & 5.40204 & 2.27523 \\
7 & 6.55616 & 2.69154 \\
8 & 7.67909 & 3.1141 \\
9 & 9.29459 & 3.68852 \\
10 & 10.4217 & 4.08574 \\
11 & 11.8255 & 4.59905 \\
12 & 13.0628 & 5.04772 \\
13 & 14.4586 & 5.55345 \\
14 & 16.0157 & 6.10734 \\
15 & 17.4963 & 6.65405 \\
20 & 24.2381 & 9.02191 \\
25 & 31.4048 & 11.6058 \\
30 & 38.5573 & 14.1547 \\
35 & 45.6409 & 16.6991 \\
40 & 53.1646 & 19.3885 \\
45 & 60.1011 & 21.8493 \\
50 & 67.254 & 24.429 \\
\hline
\end{tabular}

The Liaison Theorem: Given a group of $n \alpha$-cohesive judgments, a liaison does not exist if and only if all subgroups of cardinality (n-1) are $\alpha$-cohesive.

The existence of a liaison means that we may be able to divide a group into two subgroups whose preferences differ, and for which the geometric mean cannot be used as the representative group judgment. This is the subject of further study.

\section{Geometric Dispersion and Priority Variation}

To study the relationship that exists between the geometric dispersion of a group and the dispersion of the corresponding eigenvectors, we find the range of variability of each component of the eigenvector for given sets of group judgments. This is done by first finding the distribution of the eigenvector components for random reciprocal 
matrices whose entries are distributed according to reciprocal uniform distributions $\mathrm{RU}\left[l_{i j}, u_{i j}\right]$.

Theorem 2: For a random reciprocal matrix $X=\left(x_{i j}\right)$ with entries distributed according to a reciprocal uniform distribution, $x_{i j} \sim R U\left[\left[_{i j}, u_{i j}\right]\right.$, the components of the random variable $w=\left(w_{1}, \ldots, w_{n}\right)^{T}$ corresponding to the principal right eigenvector are distributed according to a beta, $\frac{w_{i}-\underline{w}_{i}}{\bar{w}_{i}-\underline{w}_{i}} \square \operatorname{Beta}\left(\alpha_{i}, \beta_{i}\right)$, where $\underline{w}_{i}=\min \left\{w_{i}\right\}$ and $\bar{w}_{i}=\max \left\{w_{i}\right\}$, and the principal right eigenvector of the reciprocal matrix whose entries are given by the geometric mean of its entries, $E_{G}\left[x_{i j}\right]$, is given by:

$\left(E\left[w_{1}\right], \ldots, E\left[w_{n}\right]\right)^{T}=\left(\frac{\alpha_{1}}{\alpha_{1}+\beta_{1}}\left(\bar{w}_{1}-\underline{w}_{1}\right)+\underline{w}_{1}, \cdots, \frac{\alpha_{n}}{\alpha_{n}+\beta_{n}}\left(\bar{w}_{n}-\underline{w}_{n}\right)+\underline{w}_{n}\right)^{T}$.

Let $x_{i j}=\mu_{i j} w_{i j}^{\sigma_{i j}}$ where $\mu_{i j}=\sqrt{l_{i j} u_{i j}}$ is the geometric mean and $\sigma_{i j}$ is the geometric dispersion of $x_{i j} \sim R U\left[l_{i j}, u_{i j}\right]$. By definition, $\mu_{j i}=1 / \mu_{i j}$ and $\sigma_{j i}=\sigma_{i j}$. Thus, we have $w_{j i}=1 / w_{i j}$. Let us assume that the reciprocal matrix of geometric means is consistent, i.e., $\mu_{i j} \mu_{j k}=\mu_{i k}$. Then the principal right (pr-) eigenvector of the matrix $\left(x_{i j}=\mu_{i j} w_{i j}^{\sigma_{i j}}\right)$ is given by the Hadamard product of the pr-eigenvector of the matrix $\left(\mu_{i j}\right)$, $\mu_{w}$, and the pr-eigenvector of the matrix $\left(w_{i j}^{\sigma_{i j}}\right)$. The entries of this matrix are random reciprocal uniform variables $R U\left[l_{i j} / \mu_{i j}, u_{i j} / \mu_{i j}\right]$ whose geometric dispersion is given by $\left(u_{i j} / l_{i j}\right)^{1 / 4}$. Since the geometric dispersion of the variables $x_{i j}$ and that of the variables $w_{i j}^{\sigma_{i j}}$ is the same, because $x_{i j} / \mu_{i j}=w_{i j}^{\sigma_{i j}}$, we have $\sigma_{i j}=\left(u_{i j} / l_{i j}\right)^{1 / 4}$. Thus, bounding the dispersion of the entries of the matrix $\left(w_{i j}^{\sigma_{i j}}\right)$ bounds the dispersion of the entries of the $\operatorname{matrix}\left(x_{i j}=\mu_{i j} w_{i j}^{\sigma_{i j}}\right)$.

Consider a group of five people who provide the judgments given in the following matrix:

$$
\left(\begin{array}{cccc}
1 & (2,3,4,5,6) & (1 / 2,2,1,1 / 3,4) & (3,4,1 / 2,2,8) \\
& 1 & (1,2,3,4,5) & (5,4,3,2,1) \\
& & 1 & (1 / 4,1 / 3,1,2,5) \\
& & & 1
\end{array}\right)
$$

The geometric dispersion of each group and their corresponding p-values (see Table 2 ) that the judgments $(1,3),(1,4)$ and $(3,4)$ have large geometric dispersion. This leads to large dispersion on the values of the eigenvector components (See Table 3) and a violation of Pareto Optimality. Reducing the dispersion of the judgments as in the matrix below, for example,

$$
\left(\begin{array}{cccc}
1 & (2,3,4,5,6) & (2,2,1,1,2) & (3,4,3,2,8) \\
& 1 & (1,2,3,4,5) & (5,4,3,2,1) \\
& & 1 & (1,2,1,2,5) \\
& & & 1
\end{array}\right)
$$


leads to less dispersed eigenvectors that satisfy Pareto Optimality (See Table 4).

Table 2.

Geometric Dispersions and p-values

\begin{tabular}{|c|c|c|c|c|}
\hline GD & $\begin{array}{r}1 \\
1.399306 \\
2.194046 \\
2.586241 \\
\end{array}$ & $\begin{array}{r}1.399306 \\
1 \\
1.63026 \\
1.63026 \\
\end{array}$ & $\begin{array}{r}2.194046 \\
1.63026 \\
1 \\
2.62424 \\
\end{array}$ & $\begin{array}{r}2.586241 \\
1.63026 \\
2.62424 \\
1\end{array}$ \\
\hline$p$-value & 0 & $\begin{array}{r}0.005 \\
0\end{array}$ & $\begin{array}{r}0.157 \\
0.025 \\
0\end{array}$ & $\begin{array}{r}0.3 \\
0.025 \\
0.315 \\
\end{array}$ \\
\hline
\end{tabular}

Table 3.

Individual Eigenvectors and Eigenvector of the Geometric Mean

\begin{tabular}{l|ccccc|c|}
\multicolumn{1}{c}{} & P1 & P2 & P3 & P4 & P5 & GM \\
\cline { 2 - 7 } w1 & 0.288293 & 0.460725 & 0.267140199 & 0.351129 & 0.581191 & 0.41676 \\
w2 & 0.301287 & 0.274057 & 0.283579312 & 0.281388 & 0.207615 & 0.266142 \\
w3 & 0.201647 & 0.116421 & 0.135419348 & 0.273203 & 0.136396 & 0.178548 \\
w4 & 0.208772 & 0.148798 & 0.313861141 & 0.09428 & 0.074798 & 0.138551 \\
\hline
\end{tabular}

Table 4

Individual Eigenvectors that satisfy Pareto Optimality

\begin{tabular}{l|ccccc|c|}
\multicolumn{1}{c}{} & $\mathrm{P} 1$ & $\mathrm{P} 2$ & $\mathrm{P} 3$ & $\mathrm{P} 4$ & $\mathrm{P} 5$ & $\mathrm{GM}$ \\
\cline { 2 - 7 } w1 & 0.401242 & 0.473463 & 0.439457696 & 0.438378 & 0.535109 & 0.471061 \\
w2 & 0.295662 & 0.268317 & 0.269969347 & 0.265162 & 0.227089 & 0.253814 \\
w3 & 0.187044 & 0.172147 & 0.17688459 & 0.184266 & 0.161707 & 0.176787 \\
w4 & 0.116052 & 0.086073 & 0.113688366 & 0.112194 & 0.076095 & 0.098339 \\
\hline
\end{tabular}

\section{Conclusions}

In this paper we put forth a framework to study group decision-making in the context of the AHP. A principal component of this framework is the study of the homogeneity of judgments provided by the group. We developed a new measure of the dispersion of a set of judgments from a group for a single paired comparison, and illustrated the impact that this dispersion has on the group priorities. A subject of future research is the study of the relationship between dispersions on the individual paired comparisons in the entire matrix, the consistency of judgments, the compatibility of the priority vectors and the measurement of the violation of Pareto Optimality.

\section{References}

1. Condon, E., B. Golden, and E. Wasil, Visualizing group decisions in the analytic hierarchy process. Computers and Operations Research, (2003). 30: 1435-1445.

2. Aczel, J., T.L. Saaty, Procedures for synthesizing ratio judgments. Journal of Mathematical Psychology, (1983). 27: 93-102.

3. Saaty, T.L., Fundamentals of Decision Making. Pittsburgh, PA: RWS Publications, 1994.

4. Ramanathan, R. and L.S. Ganesh, Group preference aggregation methods employed in the AHP: An evaluation and an intrinsic process for deriving member's weightages. European Journal of Operational Research, (1994). 79: 249-269. 
5. Saaty, T.L. and L.G. Vargas, The possibility of group welfare functions. Int'l Journal of Information Technology and Decision Making, (2005). 4(2): 1-10.

6. Gill, R.D., S. Johansen, A survey of product-integration with a view toward application in survival analysis. The Annals of Statistics, (1990). 18(4): 1501-1555.

7. Galambos, J., The Asymptotic Theory of Extreme Order Statistics. New York: J. Wiley, 1978. 